\title{
Computing with words and decision making
}

\author{
F. Herrera · E. Herrera-Viedma - S. Alonso • \\ F. Chiclana
}

Published online: 17 October 2009

(C) Springer Science+Business Media, LLC 2009

This special issue encompasses five papers devoted to the recent developments in the field of Computing with Words and Decision Making. The issue originated from presentations at the "8th International FLINS Conference on Computational Intelligence in Decision and Control" that was held in Madrid, Spain, September 21-24th, 2008. Every paper for the special issue was reviewed by at least two referees and finally five papers were accepted according to the referees' evaluations.

This special issue is focused in Computing with Words and Decision Making. The submissions include two review and position papers on Computing with Words and Perceptual Computing.

The first paper, "Historical Reflections and New Positions on Perceptual Computing”, by Mendel, traces the historical origins of perceptual computing. It also takes the position that interval type- 2 fuzzy sets, and not type- 1 fuzzy sets, should be used in perceptual computing. Finally, it proposes some testable guidelines for when a solution (e.g., perceptual computing) may be branded computing with words.

The second paper, "Computing with Words in Decision Making: Foundations, Trends and Prospects", reviews how the Computing with Words methodology has been used in the Decision Making field to create and enrich decision models in which the information that is provided and manipulated has a qualitative nature. Herrera et al. present an overview of the Computing with Words methodology and they

\footnotetext{
F. Herrera $(\varangle) \cdot$ E. Herrera-Viedma

Department of Computer Science and Artificial Intelligence, University of Granada, 18071 Granada, Spain

e-mail: herrera@decsai.ugr.es

S. Alonso

Department of Software Engineering, University of Granada, 18071 Granada, Spain

F. Chiclana

Centre for Computational Intelligence, De Montfort University, LE1 9BH Leicester, UK
} 
explore different linguistic computational models that have been applied to the decision making field. Additionally, they present an historical perspective of Computing with Words in decision making by examining the pioneer papers in the field along with its most recent applications. They also point out some current trends, open questions and prospects in the topic.

Due to the importance of aggregation in the Decision Making field, the development of new aggregation approaches and operators for ordinal information are currently a hot topic. In this special issue two papers about Computing with Words based aggregation are included.

In the first one, "An approach to Aggregation of Ordinal Information in MultiCriteria Multi-Person Decision Making Based on Choquet Integral of Fubini Type", Wang et al. describe an algorithm for the selection among $\mathrm{n}$ alternatives based on the evaluation of $n$ (distinct) groups of persons according to the same $m$ criteria. The evaluation of each person for each criterion is represented by a proportional ordinal 2-tuple and the overall opinion is aggregated by a pair of quantifier-guided ordered weighted averaging (OWA) aggregation and (floating) anchoring value-based ordered weighted averaging (AV-OWA) aggregation operators. The authors show how the decision function of the algorithm is a Choquet integral of the associated function of two variables (corresponding to the two aggregation processes in the algorithm) which can be accomplished alternatively by a Choquet integral of Fubini type.

The second one, "Linguistic-based voting through centered OWA operators" provides a comprehensive framework based on centered OWA operators and the 2-tuple model to enclose two linguistic-based voting systems that have been introduced in recent years: Majority Judgement and Range Voting. García-Lapresta et al. also show how to avoid some drawbacks of Majority Judgement and Range Voting by means of the use of suitable aggregation functions.

In the last few years, several applications of the different Decision Making theoretical models that use the Computing with Words methodology are being developed. In this special issue we include one of those applications.

In the paper, "A linguistic multi-criteria group decision support system for textile material fabric-hand evaluation" by Lu et al., a novel fabric hand-based textile material evaluation model is presented. As fabric hand evaluation requires considering multiple evaluation aspects/criteria by a group of evaluators, the authors propose a human-machine measure integrated fuzzy multi-criteria group decision-making method. They also develop a software tool which implements the proposed method and which is applied in fabric hand-based textile material evaluation.

Finally, as Guest Editors of this special issue, we would like to thank all the authors for their contributions and the referees for their outstanding cooperation and constructive input. 